\title{
Metastatic rectal cancer to papillary thyroid carcinoma: a case report and review of literature
}

\author{
Min Luo, Yu Huang, Yongqiang Li and Yumei Zhang* (1)
}

\begin{abstract}
Background: Tumor-to-tumor metastasis is a rare event. Rectal cancer to primary thyroid neoplasm metastasis is extremely rare. Herein, we reported a case of metastatic rectal adenocarcinoma to a papillary thyroid carcinoma. The incidence and clinicopathological characteristics of metastatic colorectal cancer to a thyroid gland neoplasm were described, and the pertinent literature was reviewed.
\end{abstract}

Case presentation: A 34-year-old female patient had curative treatment of initial rectal adenocarcinoma in 2012, and was found to have lung metastases by follow-up CT scan 3 years later. In 2018, she was found to have thyroid metastasis by imaging due to left neck pain and hoarseness. A fine-needle aspiration biopsy (FNAB) result suggested suspicious papillary thyroid carcinoma (PTC). The patient underwent a total thyroidectomy and bilateral cervical lymph nodes dissection. The histopathology of thyroidectomy specimen revealed a rectal adenocarcinoma metastatic to the thyroid concomitant with the papillary carcinoma in metastatic adenocarcinoma. The patient received levothyroxine supplementation therapy and palliative chemotherapy with irinotecan and anti-angiogenesis for the metastatic rectal adenocarcinoma. After 1 year of thyroidectomy, no newly developed lesion evidence of recurrent PTC was observed. The patient remains still alive.

Conclusion: The possibility of metastases should be considered in patients with a history of rectal cancer and with a thyroid lesion, particularly in those with ageing, hereditary nonpolyposis colorectal cancer (HNPCC) or long-term survival. The diagnosis should be histologically confirmed for the presence of both primary thyroid lesions and secondary thyroid neoplasms. Thyroidectomy may be a feasible treatment for symptomatic thyroid metastasis or thyroid cancer. we need to gain more available evidence from large or multi-center clinical data to help clinicians to diagnose rectal cancer to thyroid neoplasm metastases and evaluate treatment.

Keywords: Rectal cancer, Colorectal cancer, Papillary thyroid carcinoma, Secondary thyroid neoplasm, Tumor-totumor metastasis

\footnotetext{
* Correspondence: gxzym2019@163.com

Department of Medical Oncology, Guangxi Medical University Cancer

Hospital, No.71, Hedi road, Nanning 530021, PR China
}

(c) The Author(s). 2020 Open Access This article is licensed under a Creative Commons Attribution 4.0 International License, which permits use, sharing, adaptation, distribution and reproduction in any medium or format, as long as you give appropriate credit to the original author(s) and the source, provide a link to the Creative Commons licence, and indicate if changes were made. The images or other third party material in this article are included in the article's Creative Commons licence, unless indicated otherwise in a credit line to the material. If material is not included in the article's Creative Commons licence and your intended use is not permitted by statutory regulation or exceeds the permitted use, you will need to obtain permission directly from the copyright holder. To view a copy of this licence, visit http://creativecommons.org/licenses/by/4.0/ The Creative Commons Public Domain Dedication waiver (http://creativecommons.org/publicdomain/zero/1.0/) applies to the data made available in this article, unless otherwise stated in a credit line to the data. 


\section{Background}

Colorectal cancer (CRC), with an estimated number of over 1.8 million new cases and 881,000 cancer-related deaths worldwide in 2018, ranks the third most commonly diagnosed cancer but is the second leading cause of cancer-related death in the world [1]. Notably, $21-26 \%$ of CRC patients in the United States had distant metastasis at diagnosis and the overall survival rate in patients with metastatic colorectal cancer (mCRC) was $12 \%$ at 5 years [2]. The common sites of rectal cancer include regional lymph nodes, the liver, and the lungs, but metastasis to the thyroid gland is rare. And furthermore, rectal cancer to primary thyroid neoplasm metastasis, a tumor-totumor metastasis, is extremely rare. Up to date, only eight documented cases of mCRC to primary thyroid neoplasm metastasis were reported [3-10]. We report here a case of metastatic rectal adenocarcinoma to the papillary thyroid carcinoma and review the related literature on tumor-totumor metastasis of CRC in the thyroid gland.

\section{Case presentation}

A 34-year-old female patient had an abdominal perineal radical resection (Miles operation) of a rectal cancer in February 2012, with a normal carcinoembryonic antigen (CEA) level of $3 \mathrm{ng} / \mathrm{mL}$ (reference range: $<5.2 \mathrm{ng} / \mathrm{mL}$ ). The postoperative pathology revealed a moderately differentiated rectal adenocarcinoma, with obvious invasion to posterior vaginal wall and regional lymph node metastases (pT4bN1M0, stage IIIC, Dukes C). The patient had adjuvant chemotherapy with CapeOx (capecitabine and
Oxaliplatin) for six cycles postoperatively followed by chemoradiation. No evidence of tumor recurrence and metastasis was detected by fluorine-18-fluorodeoxyglucosepositron emission tomography integrated with computed tomography $\left({ }^{18}\right.$ F-FDG PET/CT) scan in January 2013. Her disease recurred in February 2015 when bilateral pulmonary metastasis was found by follow-up CT scan. Intermittent oral chemotherapy of capecitabine was performed at the patient's request from March 2015. In June 2017, she noted gradual enlargement of the anterior cervical bump, until she presented with left neck pain and hoarseness (in February 2018). Physical examination revealed a hard and diffuse goiter, which had a size of $3 \times 3 \mathrm{~cm}$, moved with deglutition. At this time, blood tests revealed an elevated CEA $(19 \mathrm{ng} / \mathrm{ml})$ and normal thyroid function. The neck ultrasound showed multiple heterogeneous hypoechoic nodules in the thyroid gland, particularly at the expense of the left lobe measuring $23 \times 17 \mathrm{~mm}$, and swollen bilateral cervical lymph nodes. A ${ }^{18} \mathrm{~F}$-FDG PET/CT scan revealed increased focal FDG uptake in the multiple thyroid masses (maximum standard uptake value [SUV max] 9.7), multiple cervical lymph nodes (SUV max 7.8), enlarged lymph nodes in the fifth region of mediastinum (SUV max 7.3), and multiple nodules in both sides of the lung (SUV max 12.6) (Fig. 1). There was no evidence of liver metastasis or abdominal masses. A fine-needle aspiration biopsy (FNAB) showed suspicious papillary thyroid carcinoma (PTC). The patient then underwent a total thyroidectomy and bilateral cervical lymph nodes dissection. Intraoperatively, the tumor was found to involve the left recurrent laryngeal nerve. The

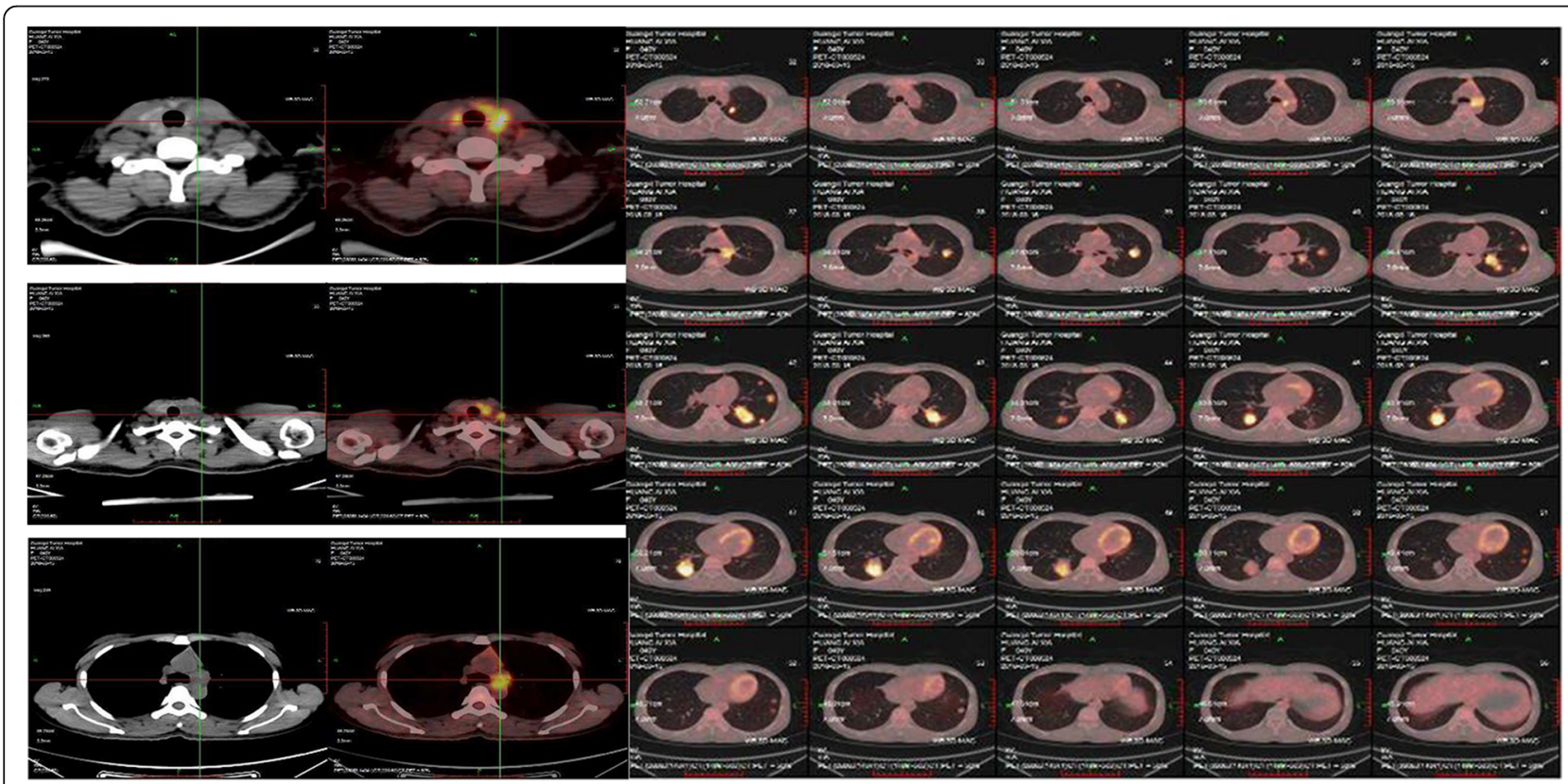

Fig. 1 The ${ }^{18}$ F-FDG PET/CT scan images showing increased focal FDG uptake in the multiple thyroid masses, cervical lymph nodes, the fifth region mediastinal lymph nodes and multiple pulmonary nodules 
histopathology of thyroidectomy specimen revealed moderately differentiated adenocarcinomas in the left and right thyroid lobes, which had the same histology as the primary rectal adenocarcinoma. In the right thyroid lobe, an incidental concomitant presence of primary papillary carcinoma (diameter $5 \mathrm{~mm}$ ) was detected in metastatic adenocarcinoma. Immunohistochemical (IHC) staining was performed using thyroid transcription factor-1 (TTF-1), paired box protein 8 (PAX8), cytokeratin 20 (CK20), caudal-related homeobox transcription factor-2 (CDX-2) and villi protein (Villin) in order to clarify the origin of the tumor. The tumor cells of metastatic rectal adenocarcinoma were positive for gastrointestinal markers CDX2, CK20 and Villin but were negative for TTF-1 and PAX8 (Fig. 2). In contrast, the tumor cells in the PTC were positive for TTF-1 and PAX8, but negative for rectal adenocarcinoma marker (Fig. 3). Histopathological and IHC examinations of the neck lymph nodes were consistent with the diagnosis of rectal adenocarcinoma metastasis. The histological diagnosis of this patient was papillary thyroid carcinoma pT1aN0M0, Stage I, and multiple metastatic rectal adenocarcinoma rT4bN1M1b, stage IVB. Due to metastatic rectal adenocarcinoma, RAS, BRAF V600E and PI3K molecular assay was performed and revealed that NRAS, BRAF V600E and PI3K was wild type but KRAS exons 2 had a mutation. Therefore, the patient was not eligible for cetuximab treatment, which was the inhibitor of the epidermal growth factor receptor (EGFR). She received levothyroxine supplementation therapy and palliative chemotherapy with irinotecan and anti-angiogenesis for the metastatic rectal adenocarcinoma.

After 1 year of thyroidectomy, no evidence showed newly developed lesion of recurrent PTC. Currently, the patient remains alive and receives Regorafenib to prevent the aggravation of pulmonary metastases.

\section{Discussion and conclusions}

Due to the fast flow of arterial blood and the high iodine and oxygen content in the thyroid gland tissue, metastasis of a cancer to the thyroid gland is a rare event in clinical practice, which comprises approximately $1.3-3 \%$ of all thyroid malignant neoplasms [11, 12]. However, autopsy series reported the overall incidence of the secondary thyroid malignancies as high as $24 \%$ in the populations with known primary cancers [13]. While metastasis of CRC to the thyroid gland is uncommon, which only accounts for about $0.1 \%$ of CRC cases [14], metastasis to an intrathyroidal primary neoplasm (i.e. tumor-to-tumor metastasis), is exceptionally uncommon. Tumor-to-tumor metastasis is defined as the metastasis of one tumor into another, including both malignant-tobenign tumor metastasis and malignant-to-malignant metastasis. The first case of tumor-to-tumor metastasis was first reported by Berent in 1902 [15]. To diagnose a tumor-to-tumor metastasis requires the following criteria as [16-19]: 1) more than one primary tumor must exist; 2) the recipient tumor must be a true benign or malignant neoplasm; 3) the donor malignancy must be a true metastasis; and 4) the exception of contiguous growth of one tumor into another adjacent tumor ("collision tumor"), embolization of tumor cells, and metastasis to the lymphatic system that were already involved by generalized lymphatic or hematological malignancy. In the present report, our patient appeared to have satisfied all these criteria based on the second pathologic examination of PTC, which was presented 6 years after curative

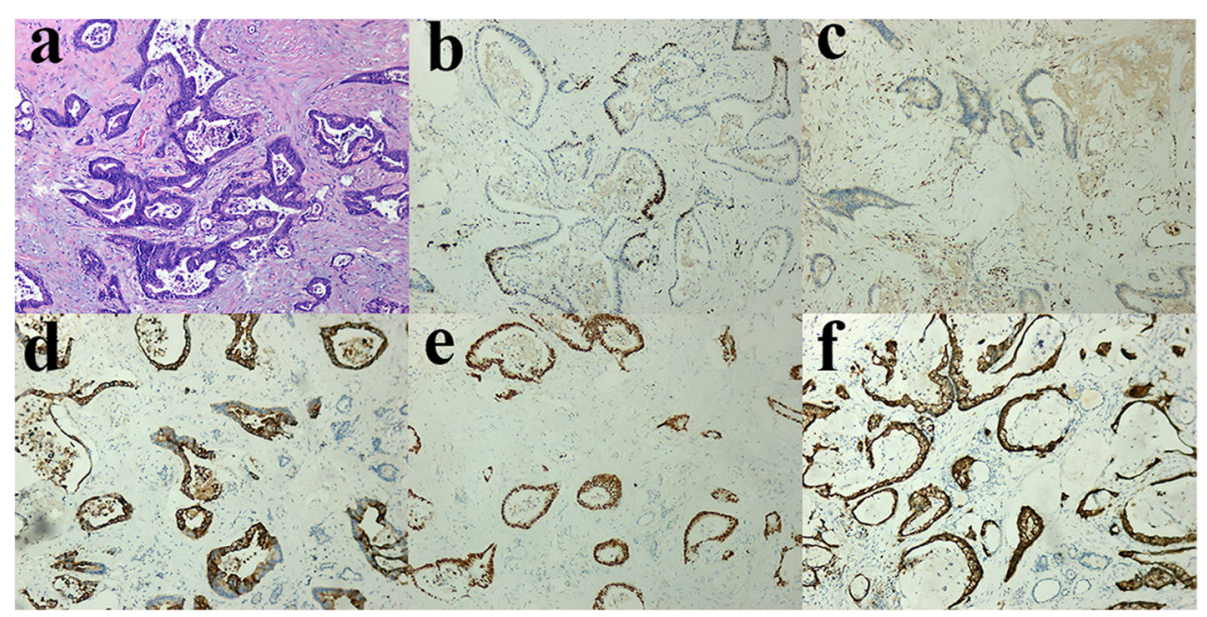

Fig. 2 Images of histopathological and immunohistochemical (IHC) showing metastatic carcinoma in the resected thyroid gland. a H\&E stain of thyroid gland detects adenocarcinoma with mucinous features, consistent with metastatic rectal adenocarcinoma. $\mathbf{b}$ Tumor cells are negative for TTF-1 staining. c Tumor cells are negative for PAX8 staining. $\mathbf{d}$ Tumor cells are positive for CK20 staining. e Tumor cells are positive for CDX-2 staining. $\mathbf{f}$ Tumor cells are positive for Villin staining. 


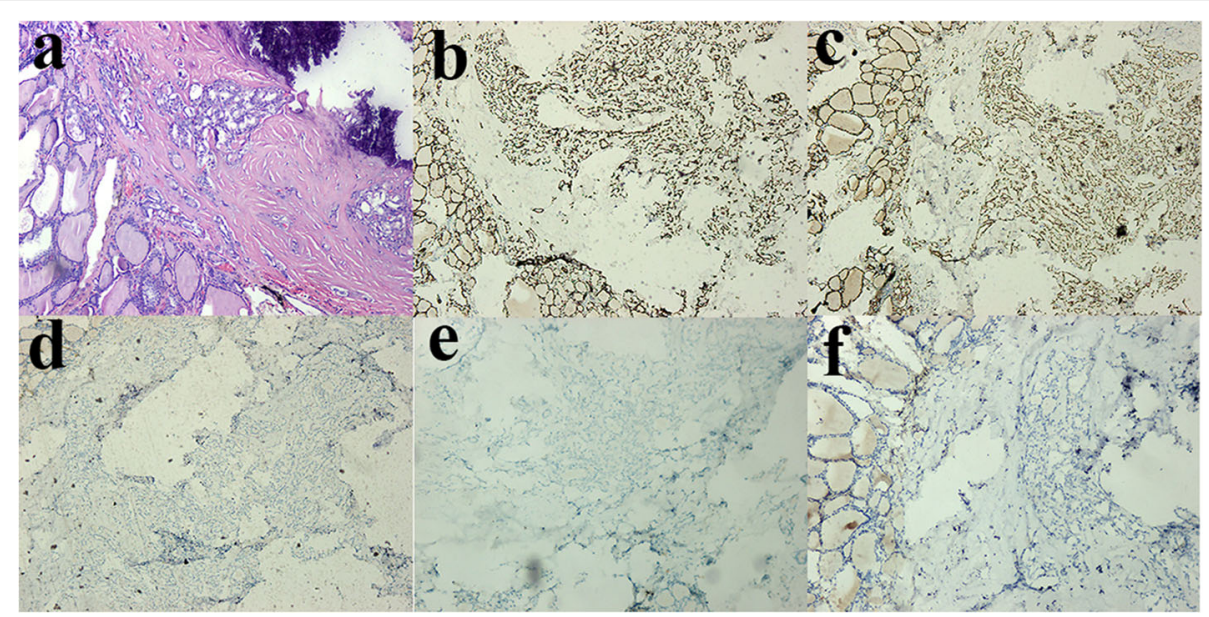

Fig. 3 Images of histopathological and immunohistochemical (IHC) showing primary papillary carcinoma in metastatic adenocarcinoma. a H\&E stain of primary papillary thyroid carcinoma. $\mathbf{b}$ Tumor cells are positive for TTF-1 staining. $\mathbf{c}$ Tumor cells are positive for PAX8 staining. $\mathbf{d}$ Tumor cells are negative for CK20 staining. eTumor cells are negative for CDX-2 staining. $\mathbf{f}$ Tumor cells are negative for Villin staining

treatment of the initial rectal adenocarcinoma, and the patient had a history of visceral metastases.

To the best of our knowledge, fewer than 50 case reports of tumor-to-tumor metastasis in which the recipient tumor was a primary thyroid neoplasm were reported in the literature. Furthermore, only eight documented cases were CRC to primary thyroid neoplasm metastasis (Table 1). Our present report is the ninth case to describe mCRC metastasis to thyroid with a PTC. The first documented case of $\mathrm{mCRC}$ to a primary thyroid neoplasm was described by Kameyama in 2000 [3]. Of the nine cases of $\mathrm{mCRC}$ to the recipient thyroid neoplasm reported, 2 the recipient thyroid tumors were follicular adenomas $[3,5], 1$ was Hürthle cell carcinoma [4], 4 were papillary carcinomas $[6,7,9], 1$ was medullary carcinoma [8], and 1 was follicular carcinoma [10]. Our literature review found that the interval between diagnosis of primary CRC and its metastasis to a thyroid neoplasm was in a range of 18 months to 9 years, mainly after curative treatment of the first diagnosis; only 1 case was detected synchronously with the primary malignancy, metastasis to a thyroid follicular adenoma as the initial presentation of a colonic adenocarcinoma [5]. Among these cases, thyroid metastasis was usually present with other sites distant metastases, especially lung metastases. Keranmu et al. reported that $81.0 \%$ of patients with mCRC to thyroid showed concomitant lung metastasis [20]. In our case, lung metastases appeared to be the first sign of hematogenous spread after 3 years from the primary diagnosis. Therefore, hematogenous dissemination can be considered to be the main pathway for rectal cancer to the thyroid gland. The "seed and soil" theory suggests that tumor or metastasis develop when viable tumor cells (the "seed") can proliferate at the favorable growth environment of specific organs or the recipient tumors (the "soil") via hematogenous dissemination $[3,15]$. Because of rich vascularity of thyroid gland, thyroid neoplasms are the common recipient tumors in tumor-totumor metastasis. The literature review also found two cases had isolated CRC to a thyroid neoplasm metastasis with a history of thyroid goiter [4, 7]. Based on the Batson's new theory of vertebral venous metastasis [21], we hypothesized that tumor cells migrate through the vertebral veins and directly enter to the thyroid without entering the thoracic and abdominal cavity, or without involving the caval vein, pulmonary vein and portal vein. In fact, the thyroid diseases are vulnerable to primary or secondary thyroid cancer growth due to cancer growth-induced decreases in blood flow and levels of oxygen and iodine, such as Hashimoto's thyroiditis. However, in the reported patients with mCRC to intra-thyroidal thyroid neoplasm metastasis, we found that the majority of patients had normal thyroid gland function. Perhaps, multinodular goiters and adenomas that change the structure, could also alter the thyroid environment so that it becomes a favorable site for metastatic colorectal cancer cells to settle and grow.

Thyroid metastases from rectal cancer may clinically present as an enlarged thyroid gland, neck mass, dysphagia, dry cough, dyspnea and hoarseness, which are similar to those of the primary thyroid malignancy. Through the literature review, we found that 5 of the all cases reported had these clinical features, and that another 4 cases were discovered as incidental findings with elevated CEA levels or during follow-up by accurate imaging examinations. CEA is one of the most widely used tumor markers, and its increase in the serum may be used for disease monitoring and evaluation of efficacy, especially for postoperative monitoring of CRC [22]. Elevated CEA levels $>5 \mathrm{ng} / \mathrm{mL}$ are helpful in detecting early recurrences and advanced stage in patients with resected 


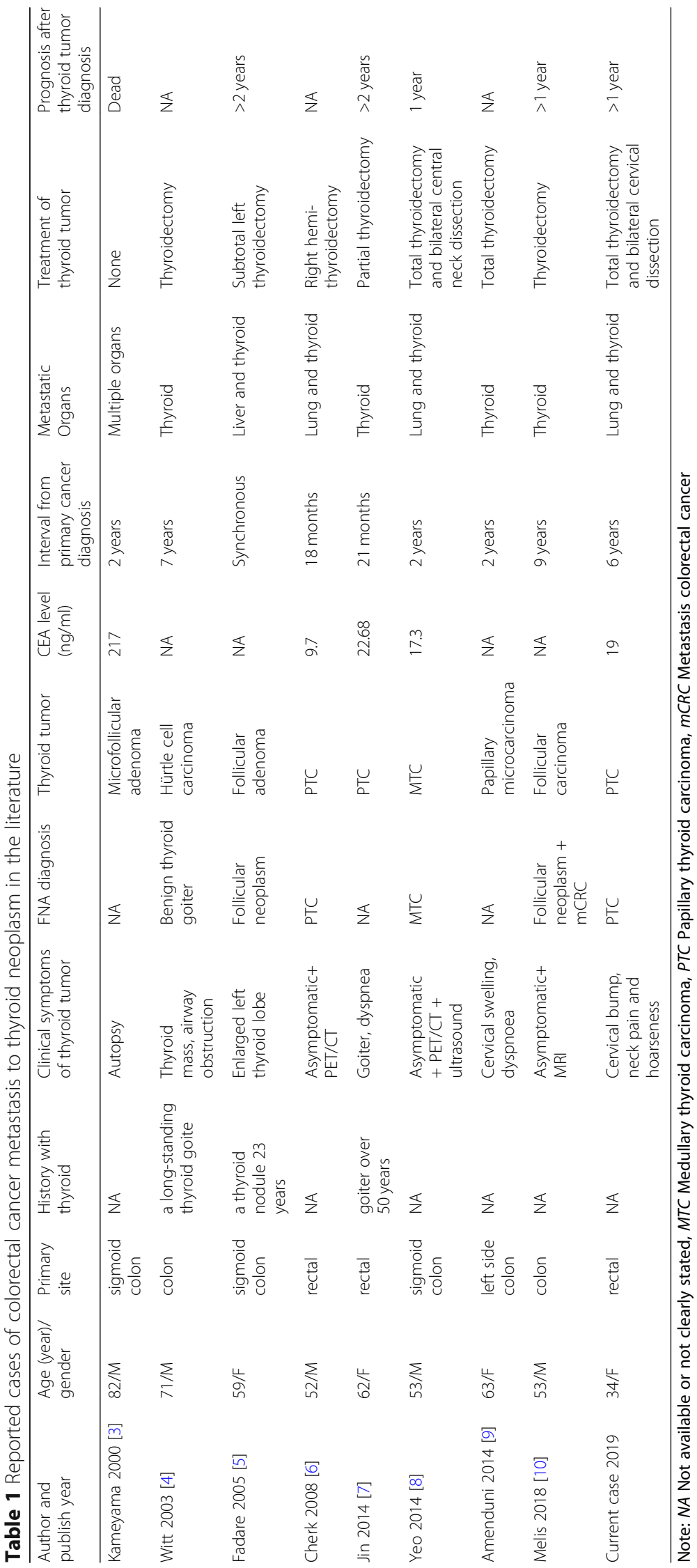


rectal cancer [23, 24]. The National Comprehensive Cancer Network (NCCN) guidelines recommended that $\mathrm{PET} / \mathrm{CT}$ scan should be more commonly used in detection of recurrent rectal cancer in patients with elevated CEA. A recent systematic review and meta-analysis of 11 studies (510 patients) found that PET/CT can be detect tumor recurrence with a sensitivity of $94.1 \%$ and a specificity of $77.2 \%$ [25]. With widely clinical use of PET/CT scan, the incidence of newly detected thyroid lesions is increasing. Previous reports described that the incidence of thyroid incidentaloma identified by PET/CT was 1.2 to $3.1 \%$ in non-thyroid disease patients and 14 to $59.8 \%$ of them were proven to be malignant, with papillary carcinomas being the common type by histopathology [26-28]. Although the accuracy of thyroid imaging has improved with the introduction of PET/CT scan, the accurate diagnosis required histology to differentiate between primary thyroid lesions and secondary thyroid neoplasms.

Presently, FNAB is the front-line method in clinic to evaluate a suspicious thyroid mass. Cytological evaluation from FNAB usually provides a rapid and reliable diagnosis of primary thyroid malignancy due to favorable sensitivity (83\%) and specificity (92\%), which is the same as metastatic thyroid neoplasm due to high accuracy (87\%) [29, 30]. In $15-30 \%$ cases, FNAB had difficulty with diagnosis, due to the issues with distinguishing the primary tumors from poorly differentiated metastatic thyroid tumors such as anaplastic thyroid carcinoma. Witt RL.et al. [4] reported a case in which FNAB diagnosed a benign thyroid goiter, but final pathologic examination indicated a colon adenocarcinoma metastatic to a thyroid Hürthle cell carcinoma with focal areas of dedifferentiated anaplastic thyroid cancer. In situations like this, IHC and molecular analysis may be useful for improving the accurate distinction between a metastatic and a primary tumor. The current study demonstrated the importance of IHC for confirming tumor-to-tumor metastasis, which showed that CDX2, CK20 and Villin were positive in metastatic rectal adenocarcinoma, and that TTF-1 and PAX8 were positive in PTC. Immunostaining for the thyroid specific biomarkers TTF-1, thyroglobulin or PAX8 can be used to differentiate between primary and secondary thyroid neoplasm. PAX 8 is an important transcription factor for thyroid organogenesis and a useful immunohistochemical marker for thyroid tumors; it is also a well-recognized marker of Müllerian tract and kidney tumors. PAX 8 can assist in distinguishing thyroid carcinomas from lung adenocarcinomas, and its sensitivity for differentiated thyroid tumors is similar to that of thyroglobulin and TTF-1 [31]. Also, PAX 8 has been reported to be positive in $74 \%$ of anaplastic thyroid carcinomas, whereas these tumors rarely express thyroglobulin and TTF-1 [32, 33]. Rectal cancer can be CK20 and CDX-2 positive, which are useful to confirm the gastrointestinal etiology, but is thyroglobulin and calcitonin negative. As in the present case, while FNAB cytology made suspicious diagnosis, histological examination of thyroidectomy confirmed it, which was further supported by specific immunohistochemical markers. Thus, specific immunohistochemical markers can be very useful in the correct diagnosis of rectal cancer to thyroid carcinomas.

Thyroidectomy is a conventional treatment for primary thyroid malignancy, is safe and may improve survival. However, thyroid metastasis appears to be an advanced disease stage, and surgery may only serve as palliative treatment. Currently, how to appropriately manage patients with thyroid metastasis remains controversial. Romero Arenas et al. [34] reported that the median overall survival (OS) was significantly longer in patients with thyroid metastasis who underwent thyroidectomy than in the nonoperative patients (34 vs. 11 months), indicating that thyroidectomy may still be beneficial for patients with thyroid metastasis. It is highly likely that the therapeutic effects of surgical management may depend on a number of factors, including the site, the stage and the performance of the primary tumor, and the symptoms caused by the thyroid mass. Of the 8 cases reported in the literature, the patients had either total or subtotal thyroidectomy. All of these patients had relieved symptoms and no one had recurrence. Russell et al. [35] recently showed that locoregional recurrence was more likely in patients with thyroid metastasis treated with subtotal thyroidectomies (13.3\%) versus total thyroidectomies (4.8\%). Even if thyroidectomy may be a palliative procedure, it can be beneficial to quality of life, reduce the incidence of locoregional recurrence and improve long-term survival. Although distant metastases are often an adverse prognosticator, thyroid metastasis may not have poor prognosis. Overall prognosis appears to be most closely associated with the advanced primary tumor. In the present case, although our patient had recurrent laryngeal nerve palsy and pulmonary metastasis of rectal adenocarcinoma, surgery relieved her clinic symptoms and controlled the local recurrence. Thus, in our case, the prognosis of this patient appeared to be determined by the patient's metastatic rectal adenocarcinoma rather than the primary thyroid malignancy.

Through the literature review, we found that, an average age of patients with CRC to thyroid tumor metastasis was 59.2 years (ranging from 34 to 85 ) and that CRC to thyroid tumor metastasis did not seem to have gender predilection. This observation was not consistent with that reported by Froylich et al. [36], who found that two thirds of the patients were female in the 34 cases of metachronous colon metastasis to the thyroid, indicating that a slight female gender predisposition might exist. In 
the series study by Papi et al. [12], the majority of the secondary lesions of the thyroid were diagnosed at a median age of 66 years, which was correlated to the increased risk of developing a malignant tumor with ageing. As reported by Kondo et al. [37], the risk of developing new primary cancer in cancer survivors, depending on age, was increased at least by $20 \%$. Lynch syndrome (also known as hereditary nonpolyposis colorectal cancer [HNPCC]) is an autosomal dominant condition that leads to increased risks for colorectal cancer, accounting for 2 to $5 \%$ of all colon and rectal cancers [38-41]. This hereditary syndrome results from a germline mutation in one of the DNA mismatch repair (MMR) genes (MLH1, MSH2, MSH6, and PMS2). Pelizzo et al. [42], revealed that both colonic and thyroid cancers were more likely to occur in association with MLH1 or MSH2 germ-line mutations in HNPCC patients. Therefore, additional thyroid monitoring should be performed in the post-treatment surveillance of patients with rectal cancer, particularly in those with ageing, HPNCC or long-term survival.

Metastatic rectal carcinoma to a primary thyroid malignancy is exceptionally uncommon. The frequency of rectal cancer to thyroid neoplasm metastasis is increasing due to the improved diagnostic technologies and long-term survival. The possibility of rectal cancer to thyroid neoplasm metastases should be considered in patients with a history of rectal cancer and with a thyroid lesion. Histological examination of thyroid specimen, histomorphology in comparison with the prior primary rectal cancer, specific immunohistochemical and molecular markers are the keys to diagnose rectal cancer to thyroid tumor metastasis. Thyroidectomy may be a feasible treatment for symptomatic thyroid metastasis or thyroid cancer which would relieve the clinical symptoms. However, these recommendations may not be translatable into clinical, for which further work is required to develop a standardized, reproducible and valid evaluation methodology. we need to gain more available evidence from large or multi-center clinical data, such as retrospective cancer center records or the National Cancer Institute's Surveillance Epidemiology and End Results (SEER). It is hoped this proposed approach will help clinicians to diagnose rectal cancer to thyroid neoplasm metastases and evaluate treatment.

\footnotetext{
Abbreviations

CRC: Colorectal cancer; mCRC: Metastatic colorectal cancer; CEA: Carcinoembryonic antigen; 18F-FDG PET/CT: Fluorine-18fluorodeoxyglucose-positron emission tomography integrated with computed tomography; SUV max: Maximum standard uptake value; FNAB: Fine-needle aspiration biopsy; PTC: Papillary thyroid carcinoma; IHC: Immunohistochemical; TTF-1: Thyroid transcription factor-1; PAX8: Paired box protein 8; CK20: Cytokeratin 20; CDX-2: Caudal-related homeobox transcription factor-2; Villin: Villi protein; EGFR: Epidermal growth factor receptor; NCCN: National Comprehensive Cancer Network; OS: Overall
}

survival; HNPCC: Hereditary nonpolyposis colorectal cancer; MMR: DNA mismatch repair

\section{Acknowledgements \\ N/A}

\section{Authors' contributions}

$M L$ wrote the manuscript and researched literature. $Y H$ and $Y L$ collected the data of the case and edited the manuscript. $Y Z$ took full responsibility for the work as a whole, including review, editing and the decision to submit and

publish the manuscript. All authors have read and approved the manuscript.

\section{Funding}

N/A

\section{Availability of data and materials}

N/A

Ethics approval and consent to participate

Ethics approval by committee was not required for this case report.

\section{Consent for publication}

Written informed consent was obtained from the patient for publication of this case report and any accompanying images. A copy of the written consent is available for review by the Editor of this journal.

\section{Competing interests}

The authors declare that they have no competing interests.

Received: 4 October 2019 Accepted: 29 April 2020

Published online: 06 May 2020

\section{References}

1. Bray F, Ferlay J, Soerjomtaram I, Siegel RL, Torre LA, Jemal A. Global Cancer statistics 2018: GLOBOCAN estimates of incidence and mortality worldwide for 36 cancers in 185 countries. CA Cancer J Clin. 2018;68(6):394-424.

2. Siegel RL, Miller KD, Jemal A. Cancer statistics, 2019. Ca-cancer J Clin. 2019; 69(1):7-34.

3. Kameyama K, Kamio N, Okita H, Ji H. Metastatic carcinoma in follicular adenoma of the thyroid gland. Pathol Res Pract. 2000;196(5):333-6.

4. Witt RL. Colonic adenocarcinoma metastatic to thyroid Hürthle cell carcinoma presenting with airway obstruction. Del Med J. 2003;75(8):285-8.

5. Fadare $\mathrm{O}$, Parkash $\mathrm{V}$, Fiedler PN, Mayerson AB, Asiyanbola B. Tumor-to-tumor metastasis to a thyroid follicular adenoma as the initial presentation of a colonic adenocarcinoma. Pathol Int. 2005;55(9):574-9.

6. Cherk MH, Moore M, Serpell J, Swain S, Topliss DJ. Metastatic colorectal cancer to a primary thyroid cancer. World J Surg Oncol. 2008;6:122.

7. Jin Y, Jiang Q, Li L, Zhao R, Xin T. Rectal cancer only metastasis to the thyroid which has a primary papillary thyroid cancer. Int J Color Dis. 2014; 29(6):769-70.

8. Yeo SJ, Kim KJ, Kim BY, Jung CH, Lee SW, Kwak JJ, Kim CH, Kang SK, Mok JO Metastasis of colon cancer to medullary thyroid carcinoma: a case report. J Korean Med Sci. 2014;29(10):1432-5.

9. Amenduni T, Carbone A, Bruno R. Precocious and isolated thyroid metastasis of colorectal adenocarcinoma and incidental thyroid papillary microcarcinoma. Endocrine. 2014;47(3):969-70.

10. Melis C, Ballaux F, Bourgain C. Curious residents of the thyroid gland: two case reports of colorectal carcinoma metastasis by fine-needle aspiration diagnosis. Acta Cytol. 2018;62:443-9.

11. Calzolari F, Sartori PV, Talarico C, Parmeggiani D, Beretta E, Pezzullo L, Bovo G, Sperlongano P, Monacelli M, Lucchini R, Misso C, Gurrado A, D'Ajello M, Uggeri F, Puxeddu E, Nasi P, Testini M, Rosato L, Barbarisio A, Avenia N. Surgical treatment of Intrathyroid metastases: preliminary results of a multicentric study. Anticancer Res. 2008;28(5B):2885-8.

12. Papi G, Fadda G, Corsello SM, Corrado S, Rossi ED, Radighieri E, Miraglia A, Carani C, Pontecorvi A. Metastases to the thyroid gland: prevalence, clinicopathological aspects and prognosis: a 10-year experience. Clin Endocrinol. 2007;66(4):565-71.

13. Berge T, Lundberg S. Cancer in Malmo 1958-1969. An autopsy study. Acta Pathol Microbiol Scand Suppl. 1977;260:1-235. 
14. Lièvre A, Leboulleux S, Boige V, Travagli JP, Dromain C, Elias D, Ducreux M, Malka D. Thyroid metastases from colorectal cancer: the Institut Gustave Roussy experience. Eur J Cancer. 2006;42(12):1756-9.

15. Berent W. Seltene metastasenbildung. Zentralbl Allg Pathol. 1902;13:406-10.

16. Campbell LV, Gilbert E, Chamberlain CR Jr, Watne AL. Metastases of cancer to cancer. Cancer. 1968;22:635-43.

17. Petraki C, Vaslamatzis M, Argyrakos T, Petraki K, Strataki M, Alexopoulos C. F S. tumor to tumor metastasis: report of two cases and review of the literature. Int J Surg Pathol. 2003;11(2):127-35.

18. Matsukuma S, Kono T, Takeo H, Hamakawa Y, Sato K. Tumor-to-tumor metastasis from lung cancer: a clinicopathological postmortem study. Virchows Arch. 2013;463(4):525-34.

19. Das S, Chaudhary N, Ang LC, Megyesi JS. Papillary thyroid carcinoma metastasizing to anaplastic meningioma: an unusual case of tumor-totumor metastasis. Brain Tumor Pathol. 2017:34(2):1-5.

20. Keranmu A, Zheng H, Wu Y, Zhao J, Xu X, Liu F, Cai S, Wang Y, Xu Y Comparative study of single-center patients with thyroid metastases from colorectal cancer and previously reported cases in the literature. World J Surg Oncol. 2017;15(1):88.

21. Batson OV. THE FUNCTION OF THE VERTEBRAL VEINS AND THEIR ROLE IN THE SPREAD OF METASTASES. Ann Surg. 1940;112(1):138-49.

22. Fakih MG, Padmanabhan A. CEA monitoring in colorectal cancer. What you should know. Oncology (Williston Park). 2006;20(6):579-87.

23. Abe S, Kawai K, Ishihara S, Nozawa H, Hata K, Kiyomatsu T, Tanaka T, Watanabe T. Prognostic impact of carcinoembryonic antigen and carbohydrate antigen 19-9 in stage IV colorectal cancer patients after R0 resection. J Surg Res. 2016;205(2):384-92.

24. Filiz Al, Sucullu I, Kurt Y, Karakas DO, Gulec B, Akin ML. Persistent high postoperative Carcinoembryonic antigen in colorectal Cancer patients- is it important? Clinics (Sao Paulo). 2009;64(4):287-94.

25. Lu YY, Chen JH, Chien CR, Chen TL, Tsai SC, Lin WY, Kao CH. Use of FDGPET or PET/CT to detect recurrent colorectal cancer in patients with elevated CEA: a systematic review and meta-analysis. Int J Color Dis. 2013; 28(8):1039-47.

26. Shi H, Yuan Z, Yang C, Zhang J, Liu C, Sun J, Ye X. Role of multi-modality functional imaging in differentiation between benign and malignant thyroid 18F-fluorodeoxyglucose incidentaloma. Clin Transl Oncol. 2019; 21(11):1561-7.

27. Yang Z, Shi W, Zhu B, Hu S, Zhang Y, Wang M, Zhang J, Yao Z, Zhang Y. Prevalence and risk of cancer of thyroid incidentaloma identified by fluorine-18 fluorodeoxyglucose positron emission tomography/computed tomography. J Otolaryngol Head Neck Surg. 2012;41(5):327-33.

28. Chen YK, Ding HJ, Chen KT, Chen YL, Liao AC, Shen YY, Su CT, Kao CH. Prevalence and risk of cancer of focal thyroid incidentaloma identified by 18F-fluorodeoxyglucose positron emission tomography for cancer screening in healthy subjects. Anticancer Res. 2005;25(2B):1421-6.

29. Pusztaszeri M, Wang H, Cibas ES, Powers CN, Bongiovanni M, Ali S, Khurana KK, Michaels PJ, Faquin WC. Fine-needle aspiration biopsy of secondary neoplasms of the thyroid gland: a multi-institutional study of 62 cases. Cancer Cytopathol. 2015;123(1):19-29.

30. Tee YY, Lowe AJ, Brand CA, Judson RT. Fine-needle aspiration may miss a third of all malignancy in palpable thyroid nodules: a comprehensive literature review. Ann Surg. 2007;246(5):714-20.

31. Ordóñez NG. Value of PAX 8 immunostaining in tumor diagnosis: a review and update. Adv Anat Pathol. 2012;19(3):140-51.

32. Bishop JA, Rajni S, Westra WH. PAX8 immunostaining of anaplastic thyroid carcinoma: a reliable means of discerning thyroid origin for undifferentiated tumors of the head and neck. Hum Pathol. 2011;42(12):1873-7.

33. Ordóñez NG, El-Naggar AK, Hickey RC, Samaan NA. Anaplastic thyroid carcinoma. Immunocytochemical study of 32 cases. Am J Clin Pathol. 1991; 96(1):15-24.

34. Romero Arenas MA, Ryu H, Lee S, Morris LF, Grubbs EG, Lee JE, Perrier ND. The Role of thyroidectomy in metastatic disease to the thyroid gland. Ann Surg Oncol. 2014;21(2):434-9.

35. Russell JO, Yan K, Burkey B, Scharpf J. Nonthyroid metastasis to the thyroid gland: case series and review with observations by primary pathology. Otolaryngol Head Neck Surg. 2016;155(6):961-8.

36. Froylich D, Shiloni E, Hazzan D. Metachronous colon metastasis to the thyroid: a case report and literature review. Case Reports in Surgery. 2013; 2013(3):1-5
37. Grundmann RT, Meyer F. Second primary malignancy among cancer survivors-epidemiology, prognosis and clinical relevance. Zentralbl Chir. 2012;137(6):565-74.

38. Lynch HT, de la Chapelle A. Hereditary colorectal cancer. N Engl J Med. 2003;348(10):919-32

39. Hampel $H$, Frankel WL, Martin E, et al. Screening for the Lynch syndrome (hereditary nonpolyposis colorectal cancer). N Engl J Med. 2005;352(18): 1851-60.

40. Hampel H, Frankel WL, Martin E, et al. Feasibility of screening for Lynch syndrome among patients with colorectal cancer. J Clin Oncol. 2008;26(35): 5783-8.

41. Hampel H. Population screening for hereditary colorectal Cancer. Surg Oncol Clin N Am. 2018;27(2):319-25.

42. Pelizzo MR, Pennelli G, Zane M, Galuppini F, Colletti PM, Merante Boschin I, Rubello D. Papillary thyroid carcinoma (PTC) in Lynch syndrome: report of two cases and discussion on Lynch syndrome behaviour and genetics. Biomed Pharmacother. 2015:74:9-16.

\section{Publisher's Note}

Springer Nature remains neutral with regard to jurisdictional claims in published maps and institutional affiliations.
Ready to submit your research? Choose BMC and benefit from:

- fast, convenient online submission

- thorough peer review by experienced researchers in your field

- rapid publication on acceptance

- support for research data, including large and complex data types

- gold Open Access which fosters wider collaboration and increased citations

- maximum visibility for your research: over $100 \mathrm{M}$ website views per year

At BMC, research is always in progress.

Learn more biomedcentral.com/submissions 